\title{
Investigation of proton damage in III-V semiconductors by optical spectroscopy
}

\author{
E. Yaccuzzi ${ }^{1,2}$ S. Khachadorian, ${ }^{3}$ S. Suárez, ${ }^{4,2}$ M. Reinoso, ${ }^{5,2}$ \\ A. R. Goñi, ${ }^{6,7}$ A. Strittmatter, ${ }^{3}$ A. Hoffmann,${ }^{3}$ and P. Giudici ${ }^{1,2}$ \\ ${ }^{1}$ Departamento Energía Solar, Centro Atómico Constituyentes, \\ Av. Gral. Paz 1499, 1650 San Martn, Argentina \\ ${ }^{2}$ CONICET, Godoy Cruz 2290 (C1425FQB) CABA, Argentina \\ ${ }^{3}$ Institut für Festkörperphysik, Technische Universität Berlin, Hardenbergstr. 36, 10623 Berlin, Germany \\ ${ }^{4}$ Laboratorio de Colisiones Atómicas, Centro Atómico Bariloche, E. Bustillo 9500, 8400 Bariloche, Argentina \\ ${ }^{5}$ Departamento Física Experimental, Centro Atómico Constituyentes, \\ Av. Gral. Paz 1499, 1650 San Martn, Argentina \\ ${ }^{6}$ ICREA, Passeig Lluís Companys 23, 08010 Barcelona, Spain \\ ${ }^{7}$ Institut de Ciència de Materials de Barcelona (ICMAB-CSIC), Campus UAB, 08193 Bellaterra, Spain
}

\begin{abstract}
We studied the damage produced by $2 \mathrm{MeV}$ proton radiation on epitaxially grown InGaP/GaAs structure by means of spatially-resolved Raman and photoluminescence (PL) spectroscopy. The irradiation was performed parallel to the sample surface in order to determine the proton penetration range in both compounds. An increase of the intensity of LO phonons and a decrease of the luminescence were observed. We associate these changes with the the creation of defects in the damage region, also responsible for the observed change of the carrier concentration in the GaAs layer, determined by the shift of the phonon-plasmon coupled mode frequency. From the spatially resolved profile of the PL and phonon intensities, we obtained the proton range in both materials and we compared them with SRIM simulations. The comparison between the experimentally obtained proton range and simulations shows a very good agreement for GaAs but a discrepancy of $20 \%$ for InGaP. This discrepancy can be explained in terms of limitations of the model to simulate the electronic orbitals and bonding structure of the simulated compound. In order to overcome this limitation we propose an increase of $40 \%$ in the electronic stopping power for InGaP.
\end{abstract}

\section{INTRODUCTION}

Multijunction solar cells based on InGaP and GaAs materials are currently the state of art for space applications due to a multiplicity of factors. However, the space is a hazardous environment containing energetic particles, which lead to degradation of the solar cell efficiency and hence, a decrease in the lifetime of satellites. Many radiation experiments have been performed towards the understanding of the changes of electrical parameters related to the degradation of solar cells [1-6]. A complementary approach to experiment is to simulate the damage on different materials produced by heavy and light ions. The widely used code SRIM (Stopping and Range of Ions in Matter) [7] models the penetration of ions when entering different target materials, together with the density of vacancies generated by them.

To gain insight in degradation of the solar cells and changes of its electric parameters, it is essential to study the impact of irradiation on its constituent materials. Irradiation damage on GaAs has already been extensively studied, including the determination of the range of protons and helium ions [8], irradiation induced changes of index of refraction [9], carrier compensation [10], and annealing effects [11], to give some examples. However, for InGaP material we found only a few reports of irradiation experiments, mostly related to the electrical parameters of the devices $[12,13]$ and few about defects in the material $[14,15]$. Although for $\mathrm{InP}$ and $\mathrm{GaP}$ compounds some works show differences between experimental results and simulations regarding the penetration of pro- tons [16], experiments on ion ranges for InGaP have not been reported yet.

In this work, we investigate the damage profile in GaAs and InGaP compounds, produced by $2 \mathrm{MeV}$ proton irradiation by means of micro-Raman and microphotoluminescence $(\mu \mathrm{PL})$ spectroscopy. In the setup geometry proposed in this work, micro-Raman spectroscopy allows for an accurate non-destructive measure of the vacancy profile $[17,18]$, whereas PL spectroscopy gives insight into the defect levels and their relation to changes in absorption and emission rates. We compare the experimental results with SRIM simulations and found a very good agreement on the proton range in GaAs, but a considerable disagreement for InGaP. The identification of shortcomings of the model and subsequent corrections of the input parameters of the code, such as the stopping power are discussed. Our work contributes to the understanding of effects of radiation on III-V compounds and proposes a systematic way for the determination of proton ranges. We can accurately determine the compound correction parameters included in the SRIM code, which will result in a better prediction of degradation of devices for space applications.

\section{EXPERIMENTAL DETAILS}

The sample, grown in a horizontal Aixtron 200 MOVPE reactor, consists of a $207 \mathrm{~nm}$ thick $\mathrm{In}_{0.499} \mathrm{Ga}_{0.501} \mathrm{P}$ layer on top of a $300 \mathrm{~nm}$ undoped $\mathrm{GaAs}$ layer, both lattice matched to the Si-doped GaAs (001) 


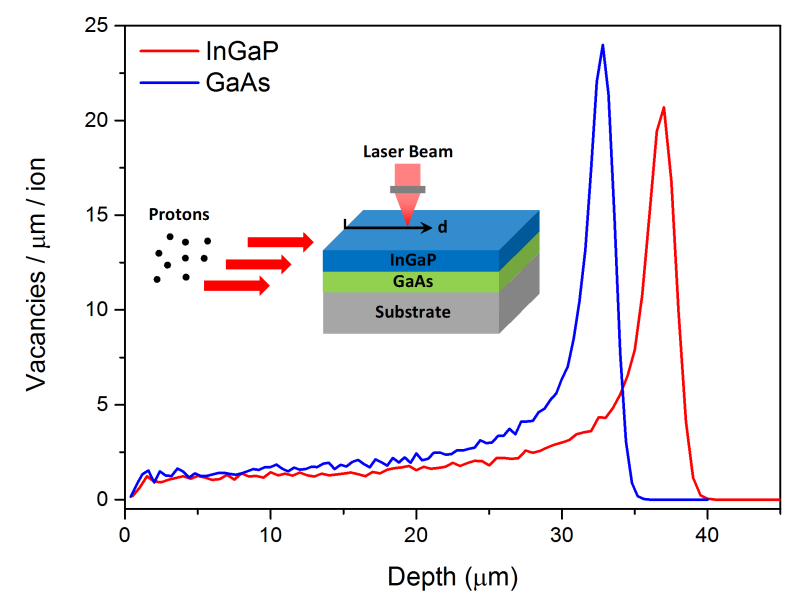

FIG. 1. Modeled vacancy profiles in InGaP and GaAs, generated by $2 \mathrm{MeV}$ protons, using the SRIM code. The inset shows the sketch of the irradiation geometry and measurement setup.

substrate with a n-doping level of $0.5-4 \times 10^{18} \mathrm{~cm}^{-3}$. Irradiation of samples in the standard configuration (with protons impacting perpendicular to the sample surface) would produce most of the damage in the substrate region. In our experimental setup the sample was irradiated parallel to its surface (see inset to Fig. 1). In this configuration, the spatially resolved measurements along the sample surface allow to study the damage profile in each layer individually.

The sample was irradiated at room temperature with protons of an energy of $2 \mathrm{MeV}$, using a Tandem accelerator [19]. The fluence was $10^{15}$ protons $\cdot \mathrm{cm}^{-2}$ and, in order to avoid radiation induced heating, the flux was kept below $2 \times 10^{12}$ protons $\cdot \mathrm{cm}^{-2} \mathrm{~s}^{-1}$. Figure 1 illustrates the vacancy profile generated by $2 \mathrm{MeV}$ protons in InGaP and GaAs, modeled using the software SRIM 2013. A mass density of $4.47 \mathrm{~g} / \mathrm{cm}^{3}$ and $5.32 \mathrm{~g} / \mathrm{cm}^{3}$ was used for InGaP and GaAs, respectively [20]. The displacement threshold energies were set to $8.8 \mathrm{eV}$ for $\mathrm{Ga}, 10.1 \mathrm{eV}$ for As, $6.7 \mathrm{eV}$ for In and $8.8 \mathrm{eV}$ for $\mathrm{P}$ [21]. The simulation yields a proton range of $36.7 \mu \mathrm{m}$ and $32.5 \mu \mathrm{m}$ for $\mathrm{InGaP}$ and GaAs, respectively, with a standard deviation (straggling) in both cases of $1.4 \mu \mathrm{m}$, and a total number of vacancies generated by each ion of 119 and 127 for InGaP and GaAs, respectively. Such a vacancy profile with a sharp peak at about the proton range is typical for stopping processes characterized by a gradual energy loss of the fast protons interacting with electrons and the final stopping of the slowed-down ions due to collisions with the atomic cores.

Raman scattering measurements were performed in backscattering geometry at room temperature using a Horiba Jobin Yvon LabRAM HR-800 spectrometer. The $514.5 \mathrm{~nm}$ line of a $\mathrm{Ar}^{+}$laser and $632.8 \mathrm{~nm}$ line of a He-Ne laser were used for excitation. A grating of $1800 \mathrm{gr} / \mathrm{mm}$ was used to gain a spectral resolution better than $1 \mathrm{~cm}^{-1}$. The laser was focused on the sample, as well as the scat- tered light was collected using a microscope objective $(\mathrm{WD}=10.6 \mathrm{~mm}, \mathrm{NA}=0.25)$ with 100 fold magnification, which results in a laser spot size of $1 \mu \mathrm{m}$. The photoluminescence (PL) measurements were performed utilizing a spectrometer with a grating of $600 \mathrm{gr} / \mathrm{mm}$ and the $514.5 \mathrm{~nm}$ line of $\mathrm{a} \mathrm{Ar}^{+}$laser for excitation.

\section{RESULTS AND DISCUSSION}

\section{A. Raman Scattering}

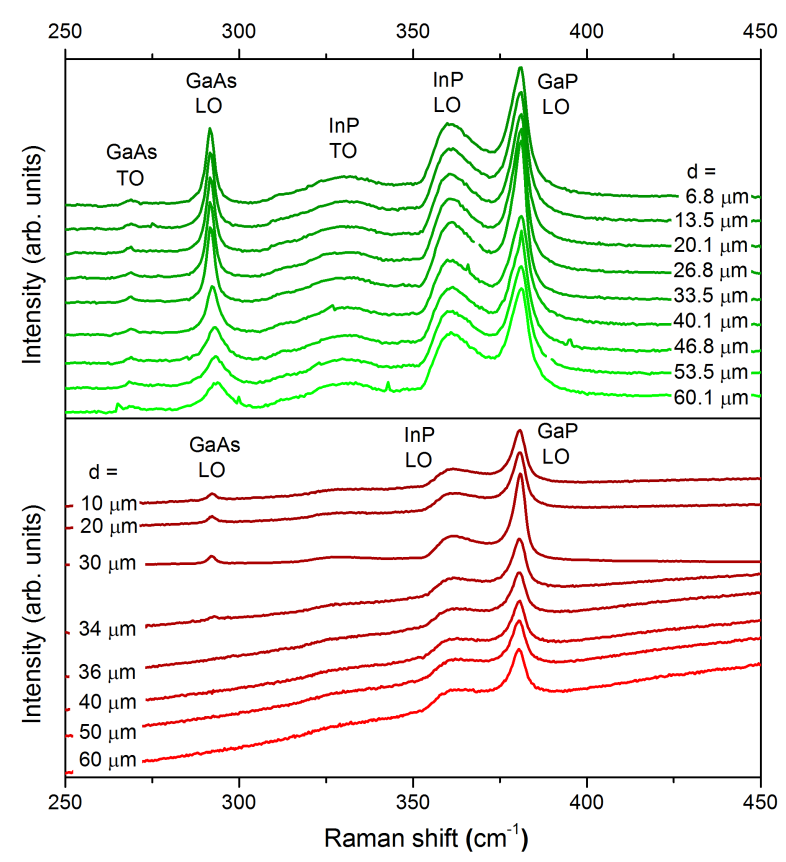

FIG. 2. Raman spectra obtained by a line scan from the edge of the sample until $60 \mu \mathrm{m}$ inwards, using a laser spot of $1 \mu \mathrm{m}$ size focused on the sample surface and an excitation wavelength of $514.5 \mathrm{~nm}$ (top) and $632.8 \mathrm{~nm}$ (bottom).

The Raman spectra in Fig. 2 show phonon modes of GaAs and InGaP by performing a line scan along the surface of the sample from the edge up to $60 \mu \mathrm{m}$ inwards, and using two different excitation energies, $632.8 \mathrm{~nm}$ and $514.5 \mathrm{~nm}$. Zinc-blende structure of III-V compounds belongs to the $\mathrm{T}_{\mathrm{d}}^{2}-\mathrm{F}-43 \mathrm{~m}$ space group, having at the $\Gamma$ point two degenerated transverse optical (TO) modes and one longitudinal optical (LO) mode. The long range electric fields generated by the polar ions leads to an increase of the LO phonon energy at the $\Gamma$-point, which is known as LO-TO splitting. Even though TO modes are forbidden for this backscattering geometry based on Raman selection rules, they can be observed due to the large numerical aperture of our setup.

The spectra exhibit the well known GaAs LO and TO modes below $300 \mathrm{~cm}^{-1}$. The peaks at $360 \mathrm{~cm}^{-1}$ and $381 \mathrm{~cm}^{-1}$ were assigned to the InP-like and GaP-like LO phonons, respectively, and the peak at $330 \mathrm{~cm}^{-1}$ to the 

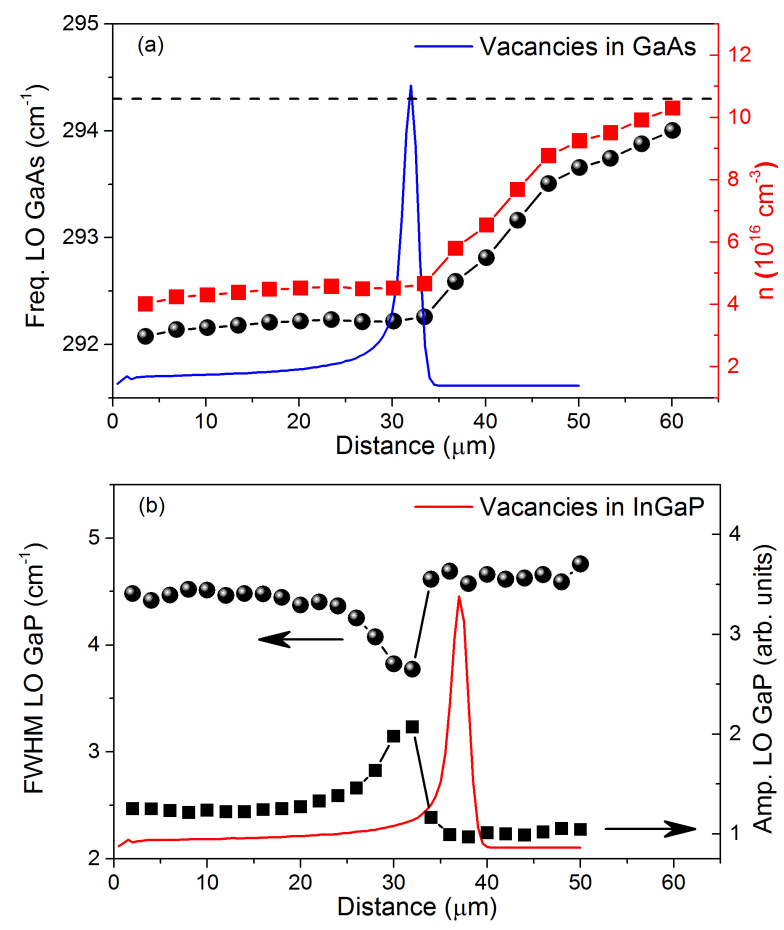

FIG. 3. Parameters of the GaAs and GaP-like LO modes obtained by fitting the Raman spectra of Fig. 2 [22]. For comparison the frequency of a non-irradiated sample (dashed lines) and the simulated vacancy profile calculated with SRIM (solid lines) are also plotted. Subfigure a) includes the carrier concentration in GaAs, calculated from the corresponding energy shift of the coupled GaAs-LO mode.

InP-like TO mode.

The line scans exhibit some noticeable changes of the Raman modes in the range of 30 to $40 \mu \mathrm{m}$, corresponding to the position where the protons have been implanted according to the SRIM simulation. By excitation with $632.8 \mathrm{~nm}$ (Fig.2 (bottom)), the GaAs LO mode $\left(292 \mathrm{~cm}^{-1}\right)$ vanishes in this region while a broad luminescence, which we assign to InGaP, arises. The $514.5 \mathrm{~nm}$ excitation (Fig.2 (top)), allows for the observation of the GaAs phonon modes in the whole region. At $30 \mu \mathrm{m}$ the LO mode loses intensity, becomes broader and shifts to higher energies. Furthermore, a clear increase of the GaP-like mode intensity at about $30 \mu \mathrm{m}$ is observed by exciting with $632.8 \mathrm{~nm}$. The InP-like LO mode shows a weaker but similar trend compared to the GaP-like mode, then it is not further discussed.

The changes in the GaAs LO mode can be explained in terms of coupling to free charge carriers: an LO phonon involves a polarization oscillation which interacts dynamically with the free carriers via the long-range Fröhlich interaction, resulting in two coupled LO-plasmon modes (LOPC) [23]. The frequency shift and shape of the peaks provide a direct insight into the carrier concentration and mobility of the materials [24, 25]. From the energy shift of the coupled LO mode we have then calculated the carrier concentration in the GaAs layer as a function of the distance from the edge. The results, included in Fig. $3 \mathrm{a}$, indicate a reduction of one order of magnitude of the electron density in the irradiated region $(0$ to $33 \mu \mathrm{m})$ [26]. This is due to the creation, by the protons, of antisite $\left(\mathrm{V}_{\mathrm{III}}\right.$ or $\left.\mathrm{III}_{\mathrm{V}}\right)$ defects in the middle of the bandgap, which act as traps for holes or electrons [27, 28].

Figure 3a also includes the simulated profile of vacancies in GaAs. The simulated implantation position of about $33 \mu \mathrm{m}$ agrees very well with the range deduced from the experiments. However, the abrupt implantation simulation profile is in clear disagreement with the gradual change of the experimental parameters. We ascribe the gradual changes to channeling [29], which is not included in the simulation code.

The effects of irradiation on the GaP-like LO-mode are illustrated in Fig. 3b. The intensity profile along the irradiation path of the GaP-like LO mode reproduce very well, not the position but the shape of the vacancies simulation, with a maximal intensity at $31 \mu \mathrm{m}$, and a minimum of the FWHM at the same position, reaching rapidly a constant value in the non irradiated region. We associate the increase of the LO intensity with an increase of the volume of the Raman scattering, related to a change in the absorption and luminescence of the materials in the irradiated region. The decrease of the phonon width is surprising, since it suggests an improvement of the quality of the crystal, in contradiction with the generation of defects by irradiation and remains an open issue. We discard an electron-phonon coupling as the origin of the observed changes of the LO mode since the frequency of the phonon remains almost unchanged within the scanned region (not shown).

\section{B. Photoluminescence}

Figure 4a shows the spatially-resolved PL spectra obtained at room temperature from 0 to $33 \mu \mathrm{m}$. Beyond $33 \mu \mathrm{m}$, the GaAs luminescence is shown in the inset. The spectra exhibit essentially two peaks around $1.43 \mathrm{eV}$ and $1.85 \mathrm{eV}$ associated with GaAs and InGaP emission, respectively. The corresponding integrated intensities are shown in Fig. 4b.

For the case of the InGaP we observe an initial decrease of the PL intensity, quenching at about $30 \mu \mathrm{m}$, followed by an abrupt increase and saturation starting from $33 \mu \mathrm{m}$. GaAs seems to be more sensitive to radiation; before $33 \mu \mathrm{m}$ there is no luminescence at all. Between 33 and $60 \mu \mathrm{m}$ the GaAs PL peak (around $1.43 \mathrm{eV}$ ) arises, increasing gradually in intensity. The reduction of the luminescence in the implantation region (up to $30 \mu \mathrm{m}$ ) can be explained by the ion-induced formation of deep levels in the band-gap, which leads to an increase of non-radiative recombination and consequently a relatively lower PL emission intensity. The critical positions of $33 \mu \mathrm{m}$ for GaAs and $30 \mu \mathrm{m}$ for InGaP indicate the place of maximal damage in each compound.

It is interesting to note that the gradual (smooth) 

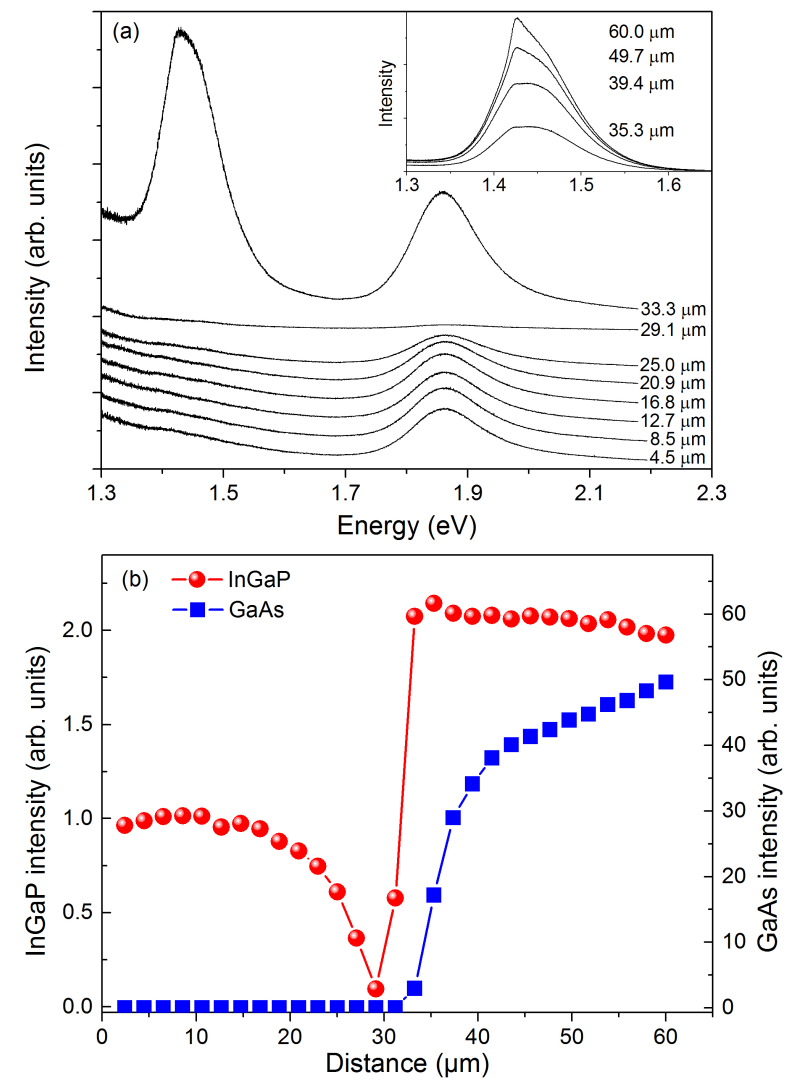

FIG. 4. a) PL line scan spectra at $300 \mathrm{~K}$ along the $\mathrm{H}^{+}$irradiation profile. b) Integrated intensity for both peaks.

change of the carrier concentration in GaAs, as determined by Raman (Fig. 3a), is also observed in the GaAs PL intensity (Fig. 4b). As mentioned before, changes in $\mathrm{PL}$ are an indication of defects creation. The monotonic increase of PL for depths several tens of microns beyond the proton range is indicative of a gradual decrease in the number of defects produced by the protons. This argument support our assumption that channeling is responsible for the smooth changes in GaAs.

\section{Simulations vs Measurements}

Concerning the proton implantation position in GaAs, we obtained a range of $33 \pm 1 \mu \mathrm{m}$, which is in excellent agreement with the $32.5 \mu \mathrm{m}$ from the simulation, and consistent with previous results [8]. On the contrary, comparing the simulation of the proton range in In$\mathrm{GaP}(37 \mu \mathrm{m})$ with the experimental results $(31 \pm 1 \mu \mathrm{m})$, we obtained an overestimation of $20 \%$ in the calculated range. Our preliminary results show that the simulation overestimates by $15 \%$ the range of InGaP in samples irradiated with $3 \mathrm{MeV}$ protons. SRIM considers separately the contribution of the cores and electronic bonds for the calculation of the stopping power. For the nuclear stopping the code approximates the compound as being amorphous using the Bragg's Rule, i.e. it considers the sum of the stopping powers of its individual elements. The electronic bonds are approximated by an electron cloud, ignoring in compounds the distribution of the electronic orbitals or bonding structure. It is well known that the electronic stopping power is relevant at high energies and when protons are slow, they interact predominantly with ions. If the incipiently observed trend of a better agreement between calculated and measured ranges is correct, this is evidence that the electronic stopping power is the origin of the problem and then ternary compounds show less accuracy than binary compounds. A systematic determination of stopping powers in III- $\mathrm{V}$ compounds is clearly necessary.

In order to overcome this limitation, the SRIM code includes a parameter named compound correction which allows for the modification only of the compound electronic stopping power. Using a correction of 1.40, the range of $2 \mathrm{MeV}$ protons in $\mathrm{InGaP}$ can be reduced from $36.7 \mu \mathrm{m}$ to $31 \mu \mathrm{m}$, consistent with our measured range. With this correction, the total number of vacancies generated per each $2 \mathrm{MeV}$ proton in $\mathrm{InGaP}$ is reduced from 119 to 95 .

\section{CONCLUSION}

In conclusion, we showed that the spatially-resolved Raman and PL spectroscopy, together with the setup proposed in this work, are powerful non-destructive methods for systematic investigations of the irradiation effects in crystals. Our experimental setup allows also for the identification of the different degradation mechanisms inside the materials and an accurate determination of ranges in different compounds. The comparison of experimental outcomes with the simulated ranges based on SRIM calculations reveals that the accuracy of the model is good for GaAs but limited for InGaP. The identification of the model shortcomings is a crucial information in the field of III-V-based optoelectronic devices for space applications.

\section{ACKNOWLEDGMENTS}

This work was supported by BEC.AR FUNDACION YPF-CONICET 2013 (Argentina) and DFG within Grant No. SFB 787. ARG thanks the Spanish Ministry of Economy and Competitiveness (MINECO) for its support through Grant No. SEV-2015-0496 in the framework of the Spanish Severo Ochoa Centre of Excellence program. 
[1] S.R. Messenger, G.P. Summers, E.A. Burke, R.J. Walters, M.A. Xapsos, Prog. Photovolt: Res. Appl. 9 (2001) 103-121.

[2] M. Yamaguchi, Sol. Energy Mater. Sol. Cells 68 (2001) 31-53.

[3] P.R. Sharps, D.J. Aiken, M.A. Stan, C.H. Thang, N. Fatemi, Prog. Photovolt: Res. Appl. 10 (2002) 383-390.

[4] M. Zazoui, M. Mbarki, A.Z. Aldin, J.C. Bourgoin, O. Gilard, G. Strobl, J. Appl. Phys. 93 (2003) 5080-5084.

[5] S. Makham, M. Zazoui, G.C. Sun, J.C. Bourgoin, Semicond. Sci. Technol. 20 (2005) 699-704.

[6] S. Sato, H. Miyamoto, M. Imaizumi, K. Shimazaki, C. Morioka, K. Kawano, T. Ohshima, Sol. Energy Mater. Sol. Cells 93 (2009) 768-773.

[7] J. F. Ziegler, M.D. Ziegler, J.P. Biersack, Nucl. Instr. and Meth. B 268 (2010) 1818-1823.

[8] J. Räisänen, E. Rauhala, Nucl. Instr. and Meth. B 93 (1994) 1-4.

[9] E. Garmire, H. Stoll, A. Yariv, R.G. Hunsperger, Appl. Phys. Lett 21 (1972) 87-88.

[10] P. Murugan, R. Kesavamoorthy, S. Amirthapandian, R. Saravanan, K. Ramachandran, N. Krishnamurthy, Physica B 315 (2002) 56-63.

[11] L.L. Abels, S. Sundaram, R.L. Schmidt, J. Comas, App. Surf. Sci. 9 (1981) 2-13.

[12] S.J. Pearton, J. M. Kuo, F. Ren, A. Katz, A.P. Perley, Appl. Phys. Lett. 59 (1991) 1467-1469.

[13] I. Danilov, J.P. de Souza, H. Boudinov, J. Bettini, M. M. G. de Carvalho, J. Appl. Phys. 92 (2002) 4261-4265.
[14] M.A. Zaidi, M. Zazoui, J.C. Bourgoin, J. Appl. Phys. 73 (1993) 7229-7231.

[15] J. Dekker, J. Oila, K. Saarinen, A. Tukiainen, W. Li, M. Pessa, J. Appl. Phys. 92 (2002) 5942-5949.

[16] C. Ascheron, Phys. Status Solidi (a) 124 (1991) 11-55.

[17] H.-Y. Kim, J.A. Freitas jr., J. Kim Year, EPL (Europhys. Lett.) 96 (2011) 26004 p1-p5.

[18] H. Huang, J. I. Dadap, O. Gaathon, I. P. Herman, R. M. Osgood, S. Bakhru, H. Bakhru, Opt. Mater. Express 3 (2013) 126-142.

[19] S. Limandri, C. Olivares, L. Rodriguez, G. Bernardi, S. Surez, Nucl. Instrum. and meth. 318 (2014) 47-50.

[20] C. Ascheron, J.P. Biersack, D. Fink, P. Goppelt, A. Manuaba, F. Paszti, N.Q. Khanh, Nucl. Instr. and Meth. B 68 (1992) 443-449.

[21] R. Bauerlein, Z. Physik 176 (1963) 498-509.

[22] Fitting was performed using peak-o-mat, C. Kristukat, 2013, http://lorentz. sourceforge.net.

[23] A. Pinczuk, G. Abstreiter, R. Trommer, M. Cardona, Sol. State Comm. 21 (1977) 959-962.

[24] G. Abstreiter, M. Cardona, A. Pinczuk, Light Scattering in Solids IV, M. Cardona, G. Güntherodt (Eds.) Springer-Verlag, Berlin, 1984.

[25] S. Ernst, A.R. Goñi, K. Syassen, M. Cardona, Phys. Rev. B 53 (1996) 1287.

[26] E.V.K. Rao, N. Duhamel, P.N. Favennec, H. L'Haridon, J. Appl. Phys. 49 (1978) 3898-3905.

[27] S.J. Pearton, Materials Science Reports 4 (1990) 313-363.

[28] J.C. Dyment, J.C. North, L.A. D'Asaro, J. Appl. Phys. 44 (1973) 207-213.

[29] R.G. Wilson, V.R. Deline, Appl. Phys. Lett. 37 (1980) 793-796. 